\title{
On the convergence of a kind of $q$-gamma operators
}

\author{
Qing-Bo Cai ${ }^{1,2}$ and Xiao-Ming Zeng ${ }^{2 *}$
}

${ }^{\text {*Correspondence: }}$

xmzeng@xmu.edu.cn

${ }^{2}$ Department of Mathematics, Xiamen University, Xiamen, 361005

P.R. China

Full list of author information is available at the end of the article

\begin{abstract}
In this paper, we introduce a kind of $q$-gamma operators based on the concept of a $q$-integral. We estimate moments of these operators and establish direct and local approximation theorems of the operators. The estimates on the rate of convergence and weighted approximation of the operators are obtained, a Voronovskaya asymptotic formula is also presented.
\end{abstract}

MSC: 41A10; 41A25; 41A36

Keywords: $q$-integral; q-gamma operators; weighted approximation; rate of convergence

\section{Introduction}

In recent years, the applications of $q$-calculus in the approximation theory is one of the main areas of research. After $q$-Bernstein polynomials were introduced by Phillips [1] in 1997, many researchers have performed studies in this field; we mention some of them [1-4].

In 2007, Karsli [5] introduced and estimated the rate of convergence for functions with derivatives of bounded variation on $[0, \infty)$ of new gamma type operators as follows:

$$
L_{n}(f ; x)=\frac{(2 n+3) ! x^{n+3}}{n !(n+2) !} \int_{0}^{\infty} \frac{t^{n}}{(x+t)^{2 n+4}} f(t) d t, \quad x>0 .
$$

In 2009, Karsli, Gupta and Izgi [6] gave an estimate of the rate of pointwise convergence of these operators (1) on a Lebesgue point of bounded variation function $f$ defined on the interval $(0, \infty)$. In 2010, Karsli and Özarslan [7] obtained some direct local and global approximation results and gave a Voronoskaya-type theorem for the operators (1). As the application of $q$-calculus in approximation theory is an active field, it seems there are no papers mentioning the $q$ analogue of these operators defined in (1). Inspired by Aral and Gupta [2], they defined a generalization of $q$-Baskakov type operators using $q$-Beta integral and obtained some important approximation properties, which motivates us to introduce the $q$ analogue of this kind of gamma operators.

Before introducing the operators, we mention certain definitions based on $q$-integers; details can be found in $[8,9]$. For any fixed real number $0<q \leq 1$ and each nonnegative integer $k$, we denote $q$-integers by $[k]_{q}$, where

$$
[k]_{q}= \begin{cases}\frac{1-q^{k}}{1-q}, & q \neq 1, \\ k, & q=1 .\end{cases}
$$

(0) 2013 Cai and Zeng; licensee Springer. This is an Open Access article distributed under the terms of the Creative Commons Attribution License (http://creativecommons.org/licenses/by/2.0), which permits unrestricted use, distribution, and reproduction in any medium, provided the original work is properly cited. 
Also, $q$-factorial and $q$-binomial coefficients are defined as follows:

$$
\begin{aligned}
& {[k]_{q} != \begin{cases}{[k]_{q}[k-1]_{q} \cdots[1]_{q},} & k=1,2, \ldots, \\
1, & k=0,\end{cases} } \\
& {\left[\begin{array}{l}
n \\
k
\end{array}\right]_{q}=\frac{[n]_{q} !}{[k]_{q} ![n-k]_{q} !} \quad(n \geq k \geq 0) .}
\end{aligned}
$$

The $q$-improper integrals are defined as (see [10])

$$
\int_{0}^{\infty / A} f(x) d_{q} x=(1-q) \sum_{-\infty}^{\infty} f\left(\frac{q^{n}}{A}\right) \frac{q^{n}}{A}, \quad A>0
$$

provided the sums converge absolutely.

The $q$-beta integral is defined by

$$
B_{q}(t ; s)=K(A ; t) \int_{0}^{\infty / A} \frac{x^{t-1}}{(1+x)_{q}^{t+s}} d_{q} x
$$

where $K(x ; t)=\frac{1}{x+1} x^{t}\left(1+\frac{1}{x}\right)_{q}^{t}(1+x)_{q}^{1-t}$ and $(a+b)_{q}^{\tau}=\prod_{j=0}^{\tau-1}\left(a+q^{j} b\right), \tau>0$.

In particular for any positive integer $m, n$,

$$
K(x, n)=q^{\frac{n(n-1)}{2}}, \quad K(x, 0)=1 \quad \text { and } \quad B_{q}(m ; n)=\frac{\Gamma_{q}(m) \Gamma_{q}(n)}{\Gamma_{q}(m+n)},
$$

where $\Gamma_{q}(t)$ is the $q$-gamma function satisfying the following functional equations:

$$
\Gamma_{q}(t+1)=[t]_{q} \Gamma_{q}(t), \quad \Gamma_{q}(1)=1
$$

(see [3]).

For $f \in C[0, \infty), q \in(0,1)$ and $n \in \mathbb{N}$, we introduce a kind of $q$-gamma operators $G_{n, q}(f ; x)$ as follows:

$$
G_{n, q}(f ; x)=\frac{[2 n+3]_{q} !\left(q^{n+\frac{3}{2}} x\right)^{n+3} q^{\frac{n(n+1)}{2}}}{[n]_{q} ![n+2]_{q} !} \int_{0}^{\infty / A} \frac{t^{n}}{\left(q^{n+\frac{3}{2}} x+t\right)_{q}^{2 n+4}} f(t) d_{q} t .
$$

Note that for $q \rightarrow 1^{-}, G_{n, 1^{-}}(f ; x)$ become the gamma operators defined in (1).

\section{Some preliminary results}

In order to obtain the approximation properties of the operators $G_{n, q}$, we need the following lemmas.

Lemma 1 For any $k \in \mathbb{N}, k \leq n+2$ and $q \in(0,1)$, we have

$$
G_{n, q}\left(t^{k} ; x\right)=\frac{[n+k]_{q} ![n-k+2]_{q} !}{[n]_{q} ![n+2]_{q} !} q^{\frac{2 k-k^{2}}{2}} x^{k} .
$$


Proof Using the properties of $q$-beta integral, we have

$$
\begin{aligned}
G_{n, q}\left(t^{k} ; x\right) & =\frac{[2 n+3]_{q} !\left(q^{n+\frac{3}{2}} x\right)^{n+3} q^{\frac{n(n+1)}{2}}}{[n]_{q} ![n+2]_{q} !} \int_{0}^{\infty / A} \frac{t^{n+k}}{\left(q^{n+\frac{3}{2}} x+t\right)_{q}^{2 n+4}} d_{q} t \\
& =\frac{x^{k}[2 n+3]_{q} ! q^{\frac{n(n+1)}{2}} q^{k\left(n+\frac{3}{2}\right)}}{[n]_{q} ![n+2]_{q} !} \int_{0}^{\infty / A} \frac{\left(\frac{t}{q^{n+\frac{3}{2}} x}\right)^{n+k}}{\left(1+\frac{t}{q^{n+\frac{3}{2}} x}\right)_{q}^{2 n+4}} d_{q}\left(\frac{t}{q^{n+\frac{3}{2}} x}\right) \\
& =\frac{x^{k}[2 n+3]_{q} ! q^{\frac{n(n+1)}{2}} q^{k\left(n+\frac{3}{2}\right)}}{[n]_{q} ![n+2]_{q} !} \frac{B_{q}(n+k+1 ; n-k+3)}{K(A ; n+k+1)} \\
& =\frac{[n+k]_{q} ![n-k+2]_{q} !}{[n]_{q} ![n+2]_{q} !} q^{\frac{2 k-k^{2}}{2}} x^{k} .
\end{aligned}
$$

Lemma 1 is proved.

Lemma 2 The following equalities hold:

$$
\begin{aligned}
& G_{n, q}(1 ; x)=1, \quad G_{n, q}(t ; x)=\frac{\sqrt{q}[n+1]_{q}}{[n+2]_{q}} x, \quad G_{n, q}\left(t^{2} ; x\right)=x^{2}, \\
& G_{n, q}\left(t^{3} ; x\right)=\frac{[n+3]_{q}}{q^{3 / 2}[n]_{q}} x^{3}, \quad G_{n, q}\left(t^{4} ; x\right)=\frac{[n+3]_{q}[n+4]_{q}}{q^{4}[n]_{q}[n-1]_{q}} x^{4}, \\
& G_{n, q}\left((t-x)^{2} ; x\right)=2 x^{2}\left(1-\frac{\sqrt{q}[n+1]_{q}}{[n+2]_{q}}\right), \\
& G_{n, q}\left((t-x)^{4} ; x\right)=\left(1+\frac{[n+3]_{q}[n+4]_{q}}{q^{4}[n]_{q}[n-1]_{q}}-\frac{4[n+3]_{q}}{q^{3 / 2}[n]_{q}}-\frac{4 \sqrt{q}[n+1]_{q}}{[n+2]_{q}}\right) x^{4} .
\end{aligned}
$$

Proof From Lemma 1, taking $k=0,1,2,3,4$, we get (6) and (7). Since $G_{n, q}\left((t-x)^{2} ; x\right)=$ $G_{n, q}\left(t^{2} ; x\right)-2 x G_{n, q}(t ; x)+x^{2}$ and $G_{n, q}\left((t-x)^{4} ; x\right)=G_{n, q}\left(t^{4} ; x\right)-4 x G_{n, q}\left(t^{3} ; x\right)+6 x^{2} G_{n, q}\left(t^{2} ; x\right)-$ $4 x^{3} G_{n, q}(t ; x)+x^{4}$, using (6), (7), we obtain (8) and (9) easily.

Remark 1 Note that for $q \rightarrow 1^{-}$, from Lemma 2, we have

$$
\begin{aligned}
& G_{n, 1^{-}}(1 ; x)=1, \quad G_{n, 1^{-}}(t ; x)=\frac{n+1}{n+2} x, \quad G_{n, 1^{-}}\left(t^{2} ; x\right)=x^{2}, \\
& G_{n, 1^{-}}\left((t-x)^{2} ; x\right)=\frac{2}{n+2} x^{2},
\end{aligned}
$$

which is the moments and central moments of the operators defined in (1).

\section{Local approximation}

In this section we establish direct and local approximation theorems in connection with the operators $G_{n, q}(f, x)$.

We denote the space of all real-valued continuous bounded functions $f$ defined on the interval $[0, \infty)$ by $C_{B}[0, \infty)$. The norm $\|\cdot\|$ on the space $C_{B}[0, \infty)$ is given by $\|f\|=$ $\sup \{|f(x)|: x \in[0, \infty)\}$. 
Further, let us consider Peetre's $K$-functional

$$
K_{2}(f, \delta)=\inf _{g \in W^{2}}\left\{\|f-g\|+\delta\left\|g^{\prime \prime}\right\|\right\},
$$

where $\delta>0$ and $W^{2}=\left\{g \in C_{B}[0, \infty): g^{\prime}, g^{\prime \prime} \in C_{B}[0, \infty)\right\}$.

For $f \in C_{B}[0, \infty)$, the modulus of continuity of second order is defined by

$$
\omega_{2}(f, \delta)=\sup _{0<h \leq \delta \in[0, \infty)} \sup _{x \in[}|f(x+2 h)-2 f(x+h)+f(x)| .
$$

By [11, p.177] there exists an absolute constant $C>0$ such that

$$
K_{2}(f, \delta) \leq C \omega_{2}(f, \sqrt{\delta}), \quad \delta>0 .
$$

Our first result is a direct local approximation theorem for the operators $G_{n, q}(f, x)$.

Theorem 1 For $q \in(0,1), x \in[0, \infty)$ and $f \in C_{B}[0, \infty)$, we have

$$
\left|G_{n, q}(f ; x)-f(x)\right| \leq C \omega_{2}\left(f ; \sqrt{\alpha_{n, q}(x)}\right)+\omega\left(f ; \beta_{n, q}(x)\right),
$$

where $C$ is a positive constant,

$$
\alpha_{n, q}(x)=\left(\frac{3}{4}-\frac{3 \sqrt{q}[n+1]_{q}}{4[n+2]_{q}}\right) x^{2}, \quad \beta_{n, q}(x)=\left(1-\frac{\sqrt{q}[n+1]_{q}}{[n+2]_{q}}\right) x .
$$

Proof Let us define the auxiliary operators

$$
\widetilde{G}_{n, q}(f ; x)=G_{n, q}(f ; x)-f\left(\frac{\sqrt{q}[n+1]_{q}}{[n+2]_{q}} x\right)+f(x),
$$

$x \in[0, \infty)$. The operators $\widetilde{G}_{n, q}(f ; x)$ are linear and preserve the linear functions

$$
\widetilde{G}_{n, q}(t-x ; x)=0
$$

(see (6)).

Let $g \in C_{B}^{2}$. By Taylor's expansion

$$
g(t)=g(x)+g^{\prime}(x)(t-x)+\int_{x}^{t}(t-u) g^{\prime \prime}(u) d u, \quad t \in[0, \infty),
$$

and (14), we get

$$
\widetilde{G}_{n, q}(g ; x)=g(x)+\widetilde{G}_{n, q}\left(\int_{x}^{t}(t-u) g^{\prime \prime}(u) d u ; x\right) .
$$

Hence, by (13) and (8), we have

$$
\begin{aligned}
& \left|\widetilde{G}_{n, q}(g ; x)-g(x)\right| \\
& \quad \leq\left|G_{n, q}\left(\int_{x}^{t}(t-u) g^{\prime \prime}(u) d u ; x\right)\right|+\left|\int_{\frac{\sqrt{q}[n+1]_{q}}{[n+2]_{q}}}^{x}\left(u-\frac{\sqrt{q}[n+1]_{q}}{[n+2]_{q}} x\right) g^{\prime \prime}(u) d u\right|
\end{aligned}
$$




$$
\begin{aligned}
& \leq G_{n, q}\left(\left|\int_{x}^{t}(t-u)\right| g^{\prime \prime}(u)|d u| ; x\right)+\int_{\frac{\sqrt{q}[n+1]_{q}}{[n+2] q}}^{x}\left|u-\frac{\sqrt{q}[n+1]_{q}}{[n+2]_{q}} x\right|\left|g^{\prime \prime}(u)\right| d u \\
& \leq\left[G_{n, q}\left((t-x)^{2} ; x\right)+\left(1-\frac{\sqrt{q}[n+1]_{q}}{[n+2]_{q}}\right)^{2} x^{2}\right]\left\|g^{\prime \prime}\right\| \\
& =\left(3-\frac{\sqrt{q}[n+1]_{q}}{[n+2]_{q}}\right)\left(1-\frac{\sqrt{q}[n+1]_{q}}{[n+2]_{q}}\right) x^{2}\left\|g^{\prime \prime}\right\| \\
& \leq 3\left(1-\frac{\sqrt{q}[n+1]_{q}}{[n+2]_{q}}\right) x^{2}\left\|g^{\prime \prime}\right\| .
\end{aligned}
$$

On the other hand, by (13), (4) and (6), we have

$$
\left|\widetilde{G}_{n, q}(f ; x)\right| \leq\left|G_{n, q}(f ; x)\right|+2\|f\| \leq\|f\| G_{n, q}(1 ; x)+2\|f\| \leq 3\|f\| .
$$

Now (13) and (15) imply

$$
\begin{aligned}
& \left|G_{n, q}(f ; x)-f(x)\right| \\
& \quad \leq\left|\widetilde{G}_{n, q}(f-g ; x)-(f-g)(x)\right|+\left|\widetilde{G}_{n, q}(g ; x)-g(x)\right|+\left|f\left(\frac{\sqrt{q}[n+1]_{q}}{[n+2]_{q}} x\right)-f(x)\right| \\
& \quad \leq 4\|f-g\|+3\left(1-\frac{\sqrt{q}[n+1]_{q}}{[n+2]_{q}}\right) x^{2}\left\|g^{\prime \prime}\right\|+\omega\left[f ;\left(1-\frac{\sqrt{q}[n+1]_{q}}{[n+2]_{q}}\right) x\right] .
\end{aligned}
$$

Hence taking infimum on the right-hand side over all $g \in W^{2}$, we get

$$
\left|G_{n, q}(f ; x)-f(x)\right| \leq 4 K_{2}\left[f ;\left(\frac{3}{4}-\frac{3 \sqrt{q}[n+1]_{q}}{4[n+2]_{q}}\right) x^{2}\right]+\omega\left[f ;\left(1-\frac{\sqrt{q}[n+1]_{q}}{[n+2]_{q}}\right) x\right] .
$$

By (10), for every $q \in(0,1)$, we have

$$
\left|G_{n, q}(f ; x)-f(x)\right| \leq C \omega_{2}\left(f ; \sqrt{\alpha_{n, q}(x)}\right)+\omega\left(f ; \beta_{n, q}(x)\right),
$$

where $\alpha_{n, q}(x)$ and $\beta_{n, q}(x)$ are defined in (12). This completes the proof of Theorem 1.

Remark 2 Let $q=\left\{q_{n}\right\}$ be a sequence satisfying $0<q_{n}<1$ and $\lim _{n} q_{n}=1$, we have $\lim _{n} \alpha_{n, q_{n}}=0$ and $\lim _{n} \beta_{n, q_{n}}(x)=0$, these give us the pointwise rate of convergence of the operators $G_{n, q_{n}}(f ; x)$ to $f(x)$.

\section{Rate of convergence}

Let $B_{x^{2}}[0, \infty)$ be the set of all functions $f$ defined on $[0, \infty)$ satisfying the condition $|f(x)| \leq M_{f}\left(1+x^{2}\right)$, where $M_{f}$ is a constant depending only on $f$. We denote the subspace of all continuous functions belonging to $B_{x^{2}}[0, \infty)$ by $C_{x^{2}}[0, \infty)$. Also, let $C_{x^{2}}^{*}[0, \infty)$ be the subspace of all functions $f \in C_{x^{2}}[0, \infty)$, for which $\lim _{x \rightarrow \infty} \frac{f(x)}{1+x^{2}}$ is finite. The norm on $C_{x^{2}}^{*}[0, \infty)$ is $\|f\|_{x^{2}}=\sup _{x \in[0, \infty)} \frac{|f(x)|}{1+x^{2}}$. We denote the usual modulus of continuity of $f$ on the closed interval $[0, a](a>0)$ by

$$
\omega_{a}(f, \delta)=\sup _{|t-x| \leq \delta x, t \in[0, a]} \sup _{|f(t)-f(x)|} \mid
$$

Obviously, for function $f \in C_{x^{2}}[0, \infty)$, the modulus of continuity $\omega_{a}(f, \delta)$ tends to zero. 
Theorem 2 Let $f \in C_{x^{2}}[0, \infty), q \in(0,1)$ and $\omega_{a+1}(f, \delta)$ be the modulus of continuity on the finite interval $[0, a+1] \subset[0, \infty)$, where $a>0$. Then we have

$$
\begin{aligned}
& \left\|G_{n, q}(f)-f\right\|_{C[0, a]} \\
& \quad \leq 12 M_{f} a^{2}\left(1+a^{2}\right)\left(1-\frac{\sqrt{q}[n+1]_{q}}{[n+2]_{q}}\right)+2 \omega_{a+1}\left(f, \sqrt{2} a \sqrt{1-\frac{\sqrt{q}[n+1]_{q}}{[n+2]_{q}}}\right) .
\end{aligned}
$$

Proof For $x \in[0, a]$ and $t>a+1$, we have

$$
|f(t)-f(x)| \leq M_{f}\left(2+x^{2}+t^{2}\right) \leq M_{f}\left[2+3 x^{2}+2(t-x)^{2}\right]
$$

hence we obtain

$$
|f(t)-f(x)| \leq 6 M_{f}\left(1+a^{2}\right)(t-x)^{2} .
$$

For $x \in[0, a]$ and $t \leq a+1$, we have

$$
|f(t)-f(x)| \leq \omega_{a+1}(f,|t-x|) \leq\left(1+\frac{|t-x|}{\delta}\right) \omega_{a+1}(f, \delta), \quad \delta>0 .
$$

From (17) and (18), we get

$$
|f(t)-f(x)| \leq 6 M_{f}\left(1+a^{2}\right)(t-x)^{2}+\left(1+\frac{|t-x|}{\delta}\right) \omega_{a+1}(f, \delta) .
$$

For $x \in[0, a]$ and $t \geq 0$, by Schwarz's inequality and Lemma 2, we have

$$
\begin{aligned}
& \left|G_{n, q}(f, x)-f(x)\right| \\
& \quad \leq G_{n, q}(|f(t)-f(x)|, x) \\
& \quad \leq 6 M_{f}\left(1+a^{2}\right) G_{n, q}\left((t-x)^{2}, x\right)+\omega_{a+1}(f, \delta)\left(1+\frac{1}{\delta} \sqrt{G_{n, q}\left((t-x)^{2}, x\right)}\right) \\
& \quad \leq 12 M_{f} a^{2}\left(1+a^{2}\right)\left(1-\frac{\sqrt{q}[n+1]_{q}}{[n+2]_{q}}\right)+\omega_{a+1}(f, \delta)\left(1+\frac{\sqrt{2} a}{\delta} \sqrt{1-\frac{\sqrt{q}[n+1]_{q}}{[n+2]_{q}}}\right) .
\end{aligned}
$$

By taking $\delta=\sqrt{2} a \sqrt{1-\frac{\sqrt{q}[n+1]_{q}}{[n+2]_{q}}}$, we get the assertion of Theorem 2 .

\section{Weighted approximation and Voronovskaya-type asymptotic formula}

Now we will discuss the weighted approximation theorem.

Theorem 3 Let the sequence $q=\left\{q_{n}\right\}$ satisfy $0<q_{n}<1$ and $q_{n} \rightarrow 1$ as $n \rightarrow \infty$, for $f \in$ $C_{x^{2}}^{*}[0, \infty)$, we have

$$
\lim _{n \rightarrow \infty}\left\|G_{n, q_{n}}(f)-f\right\|_{x^{2}}=0
$$


Proof By using the Korovkin theorem in [12], we see that it is sufficient to verify the following three conditions.

$$
\lim _{n \rightarrow \infty}\left\|G_{n, q_{n}}\left(t^{v} ; x\right)-x^{v}\right\|_{x^{2}}, \quad v=0,1,2 .
$$

Since $G_{n, q_{n}}(1 ; x)=1$ and $G_{n, q_{n}}\left(t^{2} ; x\right)=x^{2},(20)$ holds true for $v=0$ and $v=2$.

Finally, for $v=1$, we have

$$
\begin{aligned}
\left\|G_{n, q_{n}}(t ; x)-x\right\|_{x^{2}} & =\sup _{x \in[0, \infty)} \frac{\left|G_{n, q_{n}}(t ; x)-x\right|}{1+x^{2}} \\
& =\left(1-\frac{\sqrt{q_{n}}[n+1]_{q_{n}}}{[n+2]_{q_{n}}}\right) \sup _{x \in[0, \infty)} \frac{x}{1+x^{2}} \\
& \leq 1-\frac{\sqrt{q_{n}}[n+1]_{q_{n}}}{[n+2]_{q_{n}}},
\end{aligned}
$$

since $\lim _{n \rightarrow \infty} q_{n}=1$, we get $\lim _{n \rightarrow \infty} \frac{\sqrt{q}[n+1]_{q}}{[n+2]_{q}}=1$, so the condition of (21) holds for $v=1$ as $n \rightarrow \infty$, then the proof of Theorem 3 is completed.

Finally, we give a Voronovskaya-type asymptotic formula for $G_{n, q}(f ; x)$ by means of the second and fourth central moments.

Theorem 4 Let $q:=\left\{q_{n}\right\}$ be a sequence satisfying $0<q_{n}<1, \lim _{n} q_{n}=1$ and $\lim _{n} q_{n}^{n}=1$. For $f \in C_{x^{2}}^{2}[0, \infty),(f(x)$ is a twice differentiable function in $[0, \infty))$, the following equality holds

$$
\lim _{n \rightarrow \infty}[n+2]_{q}\left(G_{n, q}(f ; x)-f(x)\right)=-f^{\prime}(x) x+f^{\prime \prime}(x) x^{2}
$$

for every $x \in[0, A], A>0$.

Proof Let $x \in[0, \infty)$ be fixed. By the Taylor formula, we may write

$$
f(t)=f(x)+f^{\prime}(x)(t-x)+\frac{1}{2} f^{\prime \prime}(x)(t-x)^{2}+r(t ; x)(t-x)^{2},
$$

where $r(t ; x)$ is the Peano form of the remainder, $r(t ; x) \in C_{x^{2}}[0, \infty)$ and using L'Hopital's rule, we have

$$
\begin{aligned}
\lim _{t \rightarrow x} r(t ; x) & =\lim _{t \rightarrow x} \frac{f(t)-f(x)-f^{\prime}(x)(t-x)-\frac{1}{2} f^{\prime \prime}(x)(t-x)^{2}}{(t-x)^{2}} \\
& =\lim _{t \rightarrow x} \frac{f^{\prime}(t)-f^{\prime}(x)-f^{\prime \prime}(x)(t-x)}{2(t-x)}=\lim _{t \rightarrow x} \frac{f^{\prime \prime}(t)-f^{\prime \prime}(x)}{2}=0 .
\end{aligned}
$$

Applying $G_{n, q}(f ; x)$ to (23), we obtain

$$
\begin{aligned}
{[n+2]_{q}\left(G_{n, q}(f ; x)-f(x)\right)=} & f^{\prime}(x)[n+2]_{q} G_{n, q}(t-x ; x)+\frac{f^{\prime \prime}(x)}{2}[n+2]_{q} G_{n, q}\left((t-x)^{2} ; x\right) \\
& +[n+2]_{q} G_{n, q}\left(r(t ; x)(t-x)^{2} ; x\right) .
\end{aligned}
$$


By the Cauchy-Schwarz inequality, we have

$$
G_{n, q}\left(r(t ; x)(t-x)^{2} ; x\right) \leq \sqrt{G_{n, q}\left(r^{2}(t ; x) ; x\right)} \sqrt{G_{n, q}\left((t-x)^{4} ; x\right)} .
$$

Since $r^{2}(x ; x)=0$, then it follows from Theorem 3 that

$$
\lim _{n \rightarrow \infty} G_{n, q}\left(r^{2}(t ; x) ; x\right)=r^{2}(x ; x)=0 .
$$

Now, from (24), (25) and Lemma 2, we get immediately

$$
\lim _{n \rightarrow \infty}[n+2]_{q} G_{n, q}\left(r(t ; x)(t-x)^{2} ; x\right)=0, \quad \lim _{n \rightarrow \infty}[n+2]_{q} G_{n, q}(t-x ; x)=-x,
$$

and since $q^{n+1}=[n+2]_{q}-[n+1]_{q} \leq[n+2]_{q}-\sqrt{q}[n+1]_{q} \leq[n+2]_{q}-q[n+1]_{q}=1$, we have

$$
\begin{aligned}
\lim _{n \rightarrow \infty}[n+2]_{q} G_{n, q}\left((t-x)^{2} ; x\right) & =\lim _{n \rightarrow \infty}[n+2]_{q}\left(1-\frac{\sqrt{q}[n+1]_{q}}{[n+2]_{q}}\right) 2 x^{2} \\
& =\lim _{n \rightarrow \infty}\left([n+2]_{q}-\sqrt{q}[n+1]_{q}\right) 2 x^{2}=2 x^{2} .
\end{aligned}
$$

Theorem 4 is proved.

\section{Competing interests}

The authors declare that they have no competing interests.

\section{Authors' contributions}

QBC and XMZ carried out the molecular genetic studies, participated in the sequence alignment and drafted the manuscript. QBC and XMZ carried out the immunoassays. QBC and XMZ participated in the sequence alignment. QBC and XMZ participated in the design of the study and performed the statistical analysis. QBC and XMZ conceived of the study, and participated in its design and coordination and helped to draft the manuscript. All authors read and approved the final manuscript.

\section{Author details}

${ }^{1}$ School of Mathematics and Computer Science, Quanzhou Normal University, Quanzhou, 362000, P.R. China.

${ }^{2}$ Department of Mathematics, Xiamen University, Xiamen, 361005, P.R. China.

\section{Acknowledgements}

This work is supported by the National Natural Science Foundation of China (Grant No. 61170324), the Natural Science Foundation of Fujian Province of China (Grant No. 2010J01012) and the Project of the Educational Office of Fujian Province of China (Grant No. JK2011041).

Received: 15 November 2012 Accepted: 25 February 2013 Published: 18 March 2013

\section{References}

1. Phillips, GM: Bernstein polynomials based on the q-integers. Ann. Numer. Math. 4, 511-518 (1997)

2. Aral, A, Gupta, V: On the Durrmeyer type modification of the $q$-Baskakov type operators. Nonlinear Anal. 72(3-4), 1171-1180 (2010)

3. De Sole, A, Kac, VG: On integral representation of q-gamma and $q$-beta functions. Atti Accad. Naz. Lincei Cl. Sci. Fis. Mat. Natur. Rend. Lincei (9) Mat. Appl. 16(1), 11-29 (2005)

4. Wang, H: Korovkin-type theorem and application. J. Approx. Theory 132(2), 258-264 (2005)

5. Karsli, $\mathrm{H}$ : Rate of convergence of a new gamma type operators for functions with derivatives of bounded variation. Math. Comput. Model. 45(5-6), 617-624 (2007)

6. Karsli, H, Gupta, V, Izgi, A: Rate of point wise convergence of a new kind of gamma operators for functions of bounded variation. Appl. Math. Lett. 22, 505-510 (2009)

7. Karsli, H, Özarslan, MA: Direct local and global approximation results for operators of Gamma type. Hacet. J. Math. Stat. 39(2), 241-253 (2010)

8. Gasper, G, Rahman, M: Basic Hypergeometric Series. Encyclopedia of Mathematics and Its Applications, vol. 35. Cambridge University Press, Cambridge (1990)

9. Kac, VG, Cheung, P: Quantum Calculus. Universitext. Springer, New York (2002) 
10. Koornwinder, TH: q-special functions, a tutorial. In: Gerstenhaber, M, Stasheff, J (eds.) Deformation Theory and Quantum Groups with Applications to Mathematical Physics. Contemp. Math., vol. 134. Am. Math. Soc., Providence (1992)

11. DeVore, RA, Lorentz, GG: Constructive Approximation. Springer, Berlin (1993)

12. Gadjiev, AD: Theorems of the type of P. P. Korovkin type theorems. Math. Zametki 20(5), 781-786 (1976) (English Translation, Math. Notes 20(5-6), 996-998 (1976))

doi:10.1186/1029-242X-2013-105

Cite this article as: Cai and Zeng: On the convergence of a kind of $q$-gamma operators. Journal of Inequalities and Applications 2013 2013:105.

Submit your manuscript to a SpringerOpen ${ }^{\circ}$ journal and benefit from:

- Convenient online submission

- Rigorous peer review

- Immediate publication on acceptance

Open access: articles freely available online

- High visibility within the field

- Retaining the copyright to your article 